\title{
Avaliando a insegurança alimentar no Brasil: a questão dos efeitos não simétricos
}

\author{
Assessing food insecurity in Brazil: the issue of the non-symmetric effects
}

\author{
Ely José de Mattos (1) \\ Lorenzo Bianchi (1) \\ Camila Horst Toigo (1) \\ (1) Pontifícia Uuniversidade Católica do Rio Grande do Sul
}

\begin{abstract}
The main objective of this article is to study how a set of socioeconomics characteristics impacts the probability of occurrence of different levels of the food insecurity in Brazil. This is performed taking into consideration the informational complexity of the Brazilian Scale of Food Insecurity (EBIA). Our hypothesis, which are tested through a generalized ordered logistic model, is that the explanatory variables offer asymmetric effects regarding the different categories of food insecurity measured by the EBIA. The results confirm that hypothesis, pointing out that it is important to consider the complexity involved in the food insecurity phenomenon to discuss public policy.
\end{abstract}

\section{Keywords}

food insecurity, generalized ordered logistic model, poverty, Brazil.

JEL Codes O1, O15, I31.

\section{Resumo}

Este artigo possui como objetivo principal verificar como um grupo de características sociodemográficas afeta a probabilidade de ocorrência de diferentes niveis de insegurança alimentar no Brasil, levando em conta a complexidade informacional oferecida pela Escala Brasileira de Insegurança Alimentar (EBIA). A hipótese que avaliamos, através da proposta de aplicação de um modelo logístico ordenado generalizado, é a de que se observam efeitos assimétricos das variáveis explicativas em relação às diferentes categorias de insegurança alimentar da EBIA. Os resultados confirmam essa hipótese, alertando para a importância de se considerar a complexidade desse fenômeno na elaboração de políticas públicas nessa área.

\section{Palavras-chave}

insegurança alimentar, modelo logístico ordenado generalizado, pobreza, Brasil.

Códigos JEL O1, O15, I31. 


\section{Introdução}

De acordo com a Food and Agriculture Organization (FAO), a prevalência de subnutrição no mundo é de 10,7\% na média trienal 2014-2016. No Brasil, esse número está estimado em menos de $2,5 \%$. Ao longo dos últimos anos, a maior parte dos países monitorados atingiu o item da meta número um do Millennium Development Goals de reduzir pela metade a desnutrição entre 1990 e 2015. Apesar de o Brasil ter atingido esse objetivo meta até mesmo antes do prazo, a questão da insegurança alimentar não pode ser considerada resolvida no país (Mattos; Bagolin, 2017; IBGE, 2014; OXFAM, 2010).

Um dos aspectos que estimula a continuidade das pesquisas e debates acadêmicos e políticos sobre o tema tem relação com a própria definição de insegurança alimentar e seus consequentes desdobramentos empíricos. A FAO et al. (2017) entende que insegurança alimentar trata-se de uma situação "(...) that exists when people lack secure access to sufficient amounts of safe and nutritious food for normal growth and development and an active and healthy life" (p. 107). As estimativas da instituição são feitas de forma agregada para os países, o que denota uma perspectiva de cunho macroeconômico, que é reforçado pelas potenciais causas indicadas: "It may be caused by the unavailability of food, insufficient purchasing power, inappropriate distribution or inadequate use of food at the household level" (p. 107).

Essa abordagem, no entanto, acaba não levando em consideração uma série de aspectos particulares e subjacentes da insegurança alimentar que se enquadram num espectro conceitual e teórico mais amplo. Existe clara necessidade de se expandir esse escopo para análises pormenorizadas, considerando abordagens mais complexas e multidimensionais de aferição (Burchi; De Muro, 2016).

Uma alternativa no estudo da insegurança alimentar, que tem se consolidado ao longo dos anos, são as medidas individualizadas, onde as características objetivas e respostas pessoais compõem medidas da situação nutricional para cada pessoa ou domicílio. A escala de insegurança alimentar utilizada nos Estados Unidos foi pioneira nessa abordagem (Bickel et al. 2000). E inspirado nela o Brasil aplica, desde 2004, a Escala Brasileira de Insegurança Alimentar, EBIA (IBGE, 2014). Segundo a EBIA de 2013, $22,6 \%$ dos domicílios brasileiros experimentavam algum nível de insegu- 
rança alimentar. Ainda que o número para insegurança alimentar grave seja de 3,2\%, em linha com a magnitude que a FAO et al. (2017) informa, outros $19,4 \%$ dos domicílios enfrentam alguma restrição nutricional que precisa ser considerada.

A compreensão desse conceito mais amplo de insegurança alimentar passa, consequentemente, por sofisticar também a análise dos seus determinantes, avançando na geração de massa crítica para formulação de políticas públicas. Nesse caso, as características pessoais, familiares e domiciliares, na sua relação com a insegurança alimentar, passam a ser um importante foco analítico (Coleman-Jensen et al. 2016).

E desse contexto emerge um objeto de pesquisa em particular: as abordagens para estimar o impacto dos determinantes individuais sobre a insegurança alimentar. Assumindo que tenhamos à disposição medidas de insegurança alimentar com maior grau de complexidade e carga informacional, é importante que essa informação seja adequadamente tratada a fim de avançar no entendimento do fenômeno da forma mais completa possível.

Com base nisso, este estudo possui por objetivo principal verificar como um grupo de características sociodemográficas afeta a probabilidade de ocorrência de diferentes níveis de insegurança alimentar, levando em conta a complexidade informacional oferecida por medidas como a EBIA. A hipótese que levantamos, sustentada pelo referencial teórico, é a de que se observam efeitos assimétricos das variáveis explicativas em relação às diferentes categorias de insegurança alimentar da EBIA.

Considerando que essa escala possui quatro níveis ordinalmente dispostos - segurança alimentar e insegurança leve, moderada ou grave -, diferentes classes de modelos de probabilidade são apresentadas, sendo que em dois deles as quatro categorias são consideradas individualmente. O intuito dessa estratégia empírica é avaliar as diferenças de resultados entre os modelos e o que cada um deles oferece do ponto de vista de interpretação e análise, partindo de uma perspectiva teórica bem estabelecida.

A fundamentação teórica sobre a qual construímos o trabalho está brevemente apresentada na seção seguinte. Também apresentamos em detalhes, em seção específica, a EBIA. Posteriormente são discutidos os modelos e suas características. Ao final, temos os resultados e sua discussão. 


\section{Referencial teórico}

Em resposta ao cenário de crise de subnutrição e crescente incerteza sobre a capacidade produtiva para atender a demanda de alimentos, foi organizada pelas Nações Unidas em 1974 a primeira World Food Conference. A partir daí um background de teorias e conceitos envolvendo resiliência, segurança e acesso aos alimentos emergiu como base para abordagens que discutiam, em diferentes contextos metodológicos, operacionais e institucionais, a insegurança alimentar.

Segundo Maxwell (1996), ocorreram três mudanças de paradigmas no framework teórico e político sobre insegurança alimentar até a metade da década de 1990. A primeira delas assentou-se na unidade analítica, que passou de uma ótica macroeconômica (global e nacional) ancorada, principalmente, na oferta de níveis adequados e suficientes de alimento, para uma ótica microeconômica que focava, sobretudo, no acesso aos alimentos pelos indivíduos e domicílios. O debate inicial sobre insegurança alimentar centrou-se, então, na disponibilidade de alimentos e securitização do sistema contra riscos e identificava-se, conforme aponta Devereux e Berge (2000), que a fome era um problema relacionado à imperfeição do funcionamento do mercado de alimentos.

Após 1980, conforme Maxwell (1996), uma segunda mudança enriqueceu o debate sobre segurança alimentar: desviou-se da perspectiva de curto prazo (food-first perspective) e direcionou-se para uma perspectiva de longo prazo, preocupada com a estabilidade dos meios de vida e com a resiliência dos indivíduos (livelihoods perspective). A alternativa analítica proposta por Sen (1981a, 1981b), e que foi complementada posteriormente por outros autores como Osmani (1993), apresentava uma perspectiva de longo prazo. $\bigcirc$ constructo central para o entendimento da fome encontrou-se no conceito de entitulamento - conjunto de combinações distintas de bens, sobretudo commodities, sobre os quais um indivíduo pode obter posse e acesso no ambiente em que se encontra (Sen, 1981b).

Identificou-se que uma queda da disponibilidade de alimentos era apenas uma das possíveis causas para uma crise de fome. Havia, ainda, outras causas relacionadas aos fatores determinantes pelos quais a distribuição de alimentos ocorre em uma sociedade de trocas. A abordagem baseada nos entitulamentos preocupava-se antes com o acesso adequado aos alimentos do que com a disponibilidade garantida pela oferta suficiente de alimentos (Maxwell, 1996). 
Autores como Chambers e Conway (1991), Scoones (1998), Ellis (1998; 2000) e Ellis e Biggs (2001) trouxeram a esse debate questões importantes relacionadas à perspectiva de curto prazo. Além do acesso aos alimentos, a sustentação dos meios de vida (livelihoods) traria resiliência aos indivíduos frente aos contextos adversos, incluindo a fome. Garantidos de forma sustentável através da combinação de ativos e do acesso aos recursos indispensáveis, os meios de vida asseguravam também sustentação da vida. Assim, o seu significado mais inteligível remetia, sobretudo, à busca pela segurança, incluindo a segurança em termos alimentares.

Outros trabalhos, como os de Smith et al. (2000), Agboola e Balcilar (2012) e Maitra e Rao (2015) mostram que a pobreza, definida como situação de falta de renda e/ou ativos, está de fato relacionada à incidência de insegurança alimentar. Apontam, também, que ações na suplementação de renda têm impacto positivo no bem-estar alimentício, a exemplo do México no trabalho de Attanasio et al. (2013), do Equador em Hidrobo et al. (2014), do Canadá em Ionescu-Ittu et al. (2015).

Por fim, a última mudança de paradigma destacada por Maxwell (1996), e que está mais relacionada com a complexidade de abordagem sobre a qual este estudo está assentado, direcionou o debate para uma abordagem mais subjetiva e normativa. Esta deixou de incorporar apenas os condicionantes da privação de recursos e alimentos e passou a considerar elementos pessoais e características dos indivíduos na mensuração da segurança alimentar, como sentimentos, percepções, autoestima.

No livro Hunger and Public Action, Sen e Drèze (1989) debateram pela primeira vez a fome a partir da perspectiva da abordagem das capacitações, embora não tenham se referido, diretamente, ao conceito de segurança alimentar (Burchi; De Muro, 2016). Essa abordagem enfatiza a distinção entre meios e fins em relação ao desenvolvimento. $O$ fim maior seria a expansão das possiblidades de os indivíduos serem e fazerem coisas, desde as mais elementares, como estar nutrido adequadamente, até os as mais complexas, como ter autoestima e ser feliz (Sen, 2008; 2010; Sem; Drèze, 1989). Assim, a possibilidade de escapar da fome e dos obstáculos impostos por ela é o foco central da análise; o acesso ao alimento per se é tão somente o meio.

Com essa mudança de referência, então, é possível estabelecer um escopo analítico mais complexo para o estudo da insegurança alimentar. O trabalho de Burchi e De Muro (2016), por exemplo, faz uma sugestão de 
escopo analítico dividido em três etapas. Na primeira delas são avaliados o acesso aos alimentos e a estabilidade desse acesso. Na segunda etapa são identificadas capacitações básicas realizadas sobre as condições de acesso, como estar efetivamente nutrido, educado e saudável. Finalmente, como resultado do conjunto dos elementos anteriores, poder estar nutricionalmente seguro. Como veremos logo adiante, a ideia das escalas nutricionais é fundamentada nesse tipo de abordagem, de certa forma.

No Brasil, o debate sobre insegurança alimentar se construiu sob o exercício analítico, conceitual e de mensuração de trabalhos como os de Rocha et al. (2013), Hoffmann e Kageyama (2007), Hoffmann (2008; 2013), Belik (2003), Takagi (2006), Fávaro et al. (2007), Vianna e Segall-Corrêa (2008) e Maluf et al. (1996), que discutem o panorama e perspectivas das políticas públicas na área. O tema é especialmente interessante no Brasil também em função da trajetória do país no combate à fome, que enfileira um número considerável de projetos e programas de ação, como o Fome Zero (Mattos; Bagolin, 2017).

Hoffmann (2008), tendo por base as informações da PNAD de 2004, identificou que o determinante mais importante da insegurança alimentar é o baixo nível de renda per capita, ressaltando a importância das políticas de transferência de renda. Belik (2003) reitera que um dos maiores problemas da escassez de alimento no domicílio é a falta de renda. Hoffmann e Kageyama (2007) também identificaram que a escolaridade da pessoa de referência, a raça, o gênero e o tipo de ocupação afetam a probabilidade de reduzir a chance de o domicílio apresentar-se em situação de insegurança alimentar. Em Hoffmann (2013) as condições de domicílio (esgotamento, saneamento, água encanada) e a região brasileira, dentro da qual o domicílio era situado, também eram condicionantes significativos.

Fávaro et al. (2007) verificou a condição de segurança alimentar em famílias indígenas brasileiras. De acordo com o estudo, 75,5\% das famílias apresentaram algum grau de insegurança alimentar, sendo $22,4 \%$ insegurança leve, 32,7\% insegurança moderada e 20,4\% insegurança alimentar grave. Assim como outros autores, os determinantes indicados foram renda, escolaridade da mãe e densidade familiar.

Vianna e Segall-Corrêa (2008) também identificaram o percentual de famílias em situação de insegurança familiar em 14 municípios da Paraíba. A partir de 4.533 famílias entrevistadas constataram que apenas 47,5\% da amostra encontrava-se em situação de segurança alimentar, enquanto 
11,3\% das famílias apresentavam-se em situação de insegurança alimentar grave, $17,6 \%$ de insegurança moderada e $23,6 \%$ de insegurança leve.

De modo geral, a literatura sobre insegurança alimentar é vasta: desde trabalhos de importância teórica maior, como é o caso de Sen and Drèze (1989), Maxwell (1996) e Ellis (1998; 2000), até aplicações especializadas como Mallick and Rafi (2010), que estudam a importância do gênero dos chefes dos domicílios, ou Muraoka et al. (2018) estudando o papel do acesso à terra. E é nesse contexto profícuo que o presente trabalho pretende contribuir, discutindo o refinamento metodológico no entendimento das medidas multidimensionais de segurança alimentar, como é o caso da EBIA.

\section{A Escala Brasileira de Insegurança Alimentar (EBIA)}

Dada a variedade de aspectos que envolvem a segurança alimentar é comum encontrarmos métricas que enfatizam diferentes facetas do problema e exigem escolhas: uni ou multidimensionalidade, nível de mensuração, mensuração direta ou indireta, entre outros (Jones et al. 2013; Barret, 2010; Webb et al. 2006).

Alinhado com a perspectiva teórica multidimensional e individual de mensuração da insegurança alimentar, no contexto da terceira mudança de paradigma apontada por Maxwell (1996), este trabalho utiliza a Escala Brasileira de Segurança Alimentar (EBIA). Ela é um escala psicométrica de mensuração direta de insegurança alimentar que busca captar o acesso domiciliar a alimentos e a dimensão psicossocial da falta destes. A vantagem da utilização de uma escala psicométrica se dá na mensuração direta do fenômeno de interesse e na possibilidade de detecção de adaptações e rupturas no padrão de alimentação e outras estratégias para lidar com dificuldades na conservação deste, como ressaltam Pérez-Escamilla e Segall-Corrêa (2008).

A EBIA é baseada na escala do Departamento de Agricultura dos Estados Unidos (Bickel et al. 2000), utilizada para monitorar a insegurança alimentar nesse país. Para sua aplicação à realidade brasileira, a escala passou por um processo de validação qualitativo e quantitativo (Corrêa, 2007; Pérez-Escamilla et al. 2004). Em sua versão mais recente, a escala é composta por 14 perguntas referentes ao período de três meses anteriores à 
data de entrevista e foi realizada como um suplemento da Pesquisa Nacional por Amostra de Domicílios (PNAD) de 2013 (IBGE, 2013). As quatro primeiras são perguntas gerais, que servem para determinar a existência de algum grau de insegurança alimentar no domicílio. No caso da resposta afirmativa para alguma delas, mais dez perguntas são realizadas, sendo quatro referentes a moradores maiores de 18 anos e seis relativas a moradores menores de 18 anos, no caso da existência desses no domicílio.

Cada resposta afirmativa fornecida pelo respondente soma um ponto em um escore, indicando um maior nível de insegurança alimentar domiciliar. O somatório dessas respostas gera um escore geral que qualifica o domicílio entre quatro categorias: segurança alimentar, insegurança alimentar leve, insegurança alimentar moderada e insegurança alimentar grave. Os pontos de corte para cada categoria dependem também da existência de moradores menores de 18 anos ou não, conforme pode ser observado na Tabela 1. Essa tabela também traz uma descrição sintética de cada faixa da escala.

Tabela 1 Escala Brasileira de Segurança Alimentar

\begin{tabular}{|c|c|c|c|}
\hline \multirow[t]{2}{*}{$\begin{array}{l}\text { Classifi- } \\
\text { cação }\end{array}$} & \multicolumn{2}{|c|}{$\begin{array}{c}\text { Pontos de corte } \\
\text { para domicílios }\end{array}$} & \multirow[t]{2}{*}{ Descrição } \\
\hline & $\begin{array}{r}\text { Com me- } \\
\text { nores de } \\
18 \text { anos }\end{array}$ & $\begin{array}{r}\text { Sem me- } \\
\text { nores de } \\
18 \text { anos }\end{array}$ & \\
\hline $\begin{array}{l}\text { Segurança } \\
\text { alimentar }\end{array}$ & 0 & 0 & $\begin{array}{l}\text { A família/domicílio tem acesso regular e permanente a } \\
\text { alimentos de qualidade, em quantidade suficiente, sem } \\
\text { comprometer o acesso a outras necessidades essenciais. }\end{array}$ \\
\hline $\begin{array}{l}\text { Insegurança } \\
\text { alimentar } \\
\text { leve }\end{array}$ & $1-5$ & $1-3$ & $\begin{array}{r}\text { Preocupação ou incerteza quanto ao acesso aos ali- } \\
\text { mentos no futuro; qualidade inadequada dos alimentos } \\
\text { resultante de estratégias que visam não comprometer a } \\
\text { quantidade de alimentos. }\end{array}$ \\
\hline $\begin{array}{l}\text { Insegurança } \\
\text { alimentar } \\
\text { moderada }\end{array}$ & $6-9$ & $4-5$ & $\begin{array}{r}\text { Redução quantitativa de alimentos entre os adultos e/ou } \\
\text { ruptura nos padrões de alimentação resultante da falta de } \\
\text { alimentos entre os adultos. }\end{array}$ \\
\hline $\begin{array}{l}\text { Insegurança } \\
\text { alimentar } \\
\text { grave }\end{array}$ & $10-14$ & $6-8$ & $\begin{array}{r}\text { Redução quantitativa de alimentos entre as crianças e/ou } \\
\text { ruptura nos padrões de alimentação resultante da falta de } \\
\text { alimentos entre as crianças; fome (quando alguém fica o } \\
\text { dia inteiro sem comer por falta de dinheiro para comprar } \\
\text { alimentos). }\end{array}$ \\
\hline
\end{tabular}

Fonte: Adaptado do documento de Segurança Alimentar da PNAD (IBGE, 2013). 


\section{Dados e método}

Conforme já destacado anteriormente, a proposta deste trabalho é avaliar como um grupo de características sociodemográficas afeta a probabilidade de diferentes níveis de insegurança alimentar nos domicílios brasileiros. E a contribuição aqui apresentada é no sentido de comparar os resultados de três modelos diferentes, que lidam de modo distinto com a complexidade informacional da EBIA.

A EBIA utilizada é aquela aplicada no ano de 2013, que é a versão mais recente do suplemento de segurança alimentar oferecido pelo IBGE junto à PNAD anual. Essa pesquisa trabalha com amostragem complexa no intuito de dar conta da heterogeneidade de realidade que temos no país. Todos os cálculos realizados levaram em consideração essa estrutura de amostragem, conforme sugestão de Silva et al. (2002). A distribuição dos níveis de insegurança alimentar para a amostra considerada está na Tabela 2.

Tabela 2 Distribuição dos níveis de insegurança alimentar domiciliar na amostra considerando fatores de expansão - Brasil, 2013

\begin{tabular}{lrrrr}
\hline & Freq. abs. & \% & \% acum. \\
\hline 1 - Insegurança grave & $1,752,176$ & 2.9 & 2.9 \\
\hline - Insegurança moderada & $2,590,254$ & 4.3 & 7.2 \\
\hline 3 - Insegurança leve & $8,714,303$ & 14.5 & 21.8 \\
\hline 4 - Segurança & $46,929,186$ & 78.2 & 100.0 \\
\hline Total & $59,985,919$ & 100 & \\
\hline
\end{tabular}

Fonte: Elaborada pelos autores.

As variáveis sociodemográficas que figuram como explicativas da situação de insegurança alimentar dos domicílios estão dispostas na Tabela 3, onde também é possível visualizar as suas estatísticas descritivas. É importante ressaltar que a EBIA se refere sempre ao domicílio, e não às pessoas. Para as variáveis pessoais, estamos lidando com a pessoa cuja situação no domicílio é classificada como "pessoa de referência"1.

1 Para padronização da base foram mantidos apenas os domicílios particulares permanentes, foram excluídos indígenas, casos com questionário incompleto e também aqueles domicílios sem renda declarada. 
Tabela 3 Variáveis sociodemográficas

\begin{tabular}{|c|c|c|}
\hline & Média & Desvio-padrão \\
\hline Idade* & 48,3 & 15,9 \\
\hline Anos de Estudo* & 7,4 & 4,7 \\
\hline Renda domicilar per capita (RDPC)* & $1.120,1$ & $1.630,8$ \\
\hline Número de pessoas por cômodo* & 0,6 & 0,3 \\
\hline \multicolumn{3}{|l|}{ Gênero* (\%) } \\
\hline Feminino & 38,1 & \\
\hline Masculino & 61,9 & \\
\hline \multicolumn{3}{|l|}{ Raça* (\%) } \\
\hline Branca & 47,1 & \\
\hline Não branca & 52,9 & \\
\hline \multicolumn{3}{|l|}{ Tipo de família (\%) } \\
\hline Casal sem filhos & 19,7 & \\
\hline Casal com todos os filhos menores de 14 anos & 19,6 & \\
\hline Casal com todos os filhos de 14 anos ou mais & 17,1 & \\
\hline Casal com filhos menores de 14 anos e de 14 anos ou mais & 7,7 & \\
\hline Mãe com todos os filhos menores de 14 anos & 3,0 & \\
\hline Mãe com todos os filhos de 14 anos ou mais & 10,1 & \\
\hline Mãe com filhos menores de 14 anos e de 14 anos ou mais & 1,9 & \\
\hline Outros tipos de família & 20,7 & \\
\hline \multicolumn{3}{|l|}{ Ocupação* (\%) } \\
\hline Com carteira ou emprego formal ${ }^{1}$ & 33,0 & \\
\hline Desocupado & 31,7 & \\
\hline Sem carteira & 11,2 & \\
\hline Conta própria & 17,8 & \\
\hline Empregador & 3,6 & \\
\hline Não remunerado & 0,2 & \\
\hline Próprio consumo/uso & 2,4 & \\
\hline \multicolumn{3}{|l|}{ Atividades do domicílio² (\%) } \\
\hline Agrícola & 8,6 & \\
\hline Pluriativo $^{3}$ & 4,6 & \\
\hline Não agrícola & 70,7 & \\
\hline Desocupado & 16,1 & \\
\hline
\end{tabular}


Tabela 3 (continuação)

\begin{tabular}{|c|c|c|}
\hline & Média & Desvio-padrão \\
\hline \multicolumn{3}{|l|}{ Região* (\%) } \\
\hline Sudeste & 43,9 & \\
\hline Nordeste & 25,4 & \\
\hline Norte & 7,2 & \\
\hline Sul & 15,7 & \\
\hline Centro-Oeste & 7,8 & \\
\hline \multicolumn{3}{|c|}{ Condições de habitação ${ }^{4}(\%)$} \\
\hline Até um item & 2,7 & \\
\hline Dois itens & 20,8 & \\
\hline Três itens & 76,5 & \\
\hline \multicolumn{3}{|l|}{ Situação censitária (\%) } \\
\hline Rural & 13,1 & \\
\hline Urbano & 86,9 & \\
\hline Número de observações & 107.015 & \\
\hline
\end{tabular}

Fonte: Elaborada pelos autores. Dados da PNAD 2013.

* Refere-se à pessoa de referência. ${ }^{1}$ Essa categoria contempla empregado com carteira de trabalho assinada, militar, funcionário público estatutário e trabalhador doméstico com carteira assinada. ${ }^{2}$ Atividade principal do empreendimento do trabalho principal, considerando todas as pessoas do domicílio. ${ }^{3} O$ domicilio é considerado pluriativo se conta com moradores trabalhando em atividades agrícolas e não agrícolas ao mesmo tempo. ${ }^{4}$ Considera os seguintes itens: água encanada, esgotamento sanitário e energia elétrica.

Esse conjunto de variáveis, que é relativamente comum na literatura sobre o tema, compreende aquelas mais próximas da abordagem teórica multidimensional que a PNAD oferece. Boa parte dessas variáveis já apareceram, por exemplo, em Hoffmann (2013), Burchi e De Muro (2016) e Bhalla et al. (2018). Elas serão as variáveis explicativas dos três modelos estimados.

Os modelos de probabilidade com variáveis dependentes categóricas podem ser apresentados em três grandes tipos: binários, multinomiais e categóricos ordinais. O primeiro é o já tradicional modelo logístico de probabilidade, que é utilizado em uma ampla gama de aplicações com variáveis dependentes no formato $(0,1)$. Os modelos multinomiais lidam com variáveis dependentes que trazem mais de duas categorias as quais, apesar de diferentes entre si, não guardam qualquer ordem hierárquica. O terceiro tipo é utilizado em situações com mais de duas categorias diferentes entre 
si e onde exista ordem hierárquica observável - modelos aplicados sobre escala Lickert, por exemplo.

A EBIA é apresentada de modo categórico, pois são quatro níveis que vão de segurança alimentar até insegurança alimentar grave. Entre as categorias, existe uma ordem constituída, de algo bom para algo ruim, com resultados intermediários. Então, neste trabalho, vamos apresentar duas classes de modelos: binário e ordinal. Vamos partir da seguinte expressão geral dos modelos logísticos de probabilidade:

$$
P\left(Y_{i}>j\right)=g\left(X \beta_{j}\right)=\frac{\exp \left(\alpha_{j}+X_{i} \beta_{j}\right)}{1+\exp \left(\alpha_{j}+X_{i} \beta_{j}\right)}, \quad j=1,2, \ldots, M-1
$$

onde $M$ é o número de categorias da variável dependente, $\alpha$ e $\beta$ os coeficientes estimados, e $X$ o vetor de variáveis explicativas (Williams, 2006).

O modelo binário, bastante usual nessa literatura, terá sua variável dependente construída da seguinte forma: segurança alimentar e insegurança alimentar leve formam uma categoria, contra insegurança alimentar moderada e grave. Nesse caso, tome a equação (1) no caso especial de apenas duas categorias $(M=2)$. $\bigcirc$ resultado são estimativas de probabilidade com redução na complexidade informacional da EBIA, uma vez que duas classes são colapsadas. Iremos estimar esse modelo para que seja o ponto de referência analítica inicial.

Em um segundo modelo, propomos um modelo logístico ordenado. Nesse caso, a expressão (1) torna-se ligeiramente diferente:

$$
P\left(Y_{i}>j\right)=g(X \beta)=\frac{\exp \left(\alpha_{j}+X_{i} \beta\right)}{1+\exp \left(\alpha_{j}+X_{i} \beta\right)}, \quad j=1,2, \ldots, M-1
$$

Nessa equação, os coeficientes $\beta$ não dependem da categoria da variável explicada $Y$. Ou seja, existe apenas um coeficiente angular para cada variável explicativa na equação estimada, sendo que as probabilidades para cada categoria variam apenas em função dos coeficientes de nível $\left(\alpha_{j}\right)$. Isso implica a necessidade de garantir a hipótese do paralelismo, que assegura essa igualdade de coeficientes. $O$ teste de Brant faz essa avaliação (Brant, 1990).

No entanto, a literatura tem alertado que essa hipótese é bastante forte, sendo frequentemente violada - e seguidamente a violação é ignora- 
da nos estudos, o que compromete as conclusões (Williams, 2006; 2016). A nossa hipótese inicial, dada a complexidade dos dados, é que não conseguiríamos garanti-la, de modo que outra alternativa de modelo deveria ser testada.

Por fim, no intuito de levar em consideração o que Fullerton e Dixon (2010) chamam de efeitos assimétricos escondidos quando se colapsam variáveis dependentes categóricas, apresentamos o modelo logístico ordenado generalizado (Fu, 1998; Williams, 2006). ${ }^{2}$ Esse modelo, que é descrito pela expressão (1), apresenta uma solução à restrição da hipótese do paralelismo ao permitir que cada coeficiente $\beta_{j}$ seja testado para ela de forma individual. No caso de o paralelismo ser garantido para o coeficiente de determinada variável $X_{i}$, ele é utilizado uniformemente; caso não seja, são estimados diferentes $\beta_{j}$ para cada categoria $j$. Esse procedimento é operacionalizado através da estimação simultânea de $k-1$ modelos logísticos cumulativos, sendo $k$ o número de categorias da variável dependente.

Com os resultados para os três modelos, pretendemos propor a discussão sobre como os diferentes métodos são capazes de agregar a complexidade informacional contida na EBIA, oferecendo diferentes perspectivas sobre o fenômeno. Além disso, também incorporamos uma discussão sobre os resultados, propriamente, associando-os a aqueles já encontrados na literatura.

\section{Resultados e discussão}

O primeiro modelo estimado é o logit tradicional. Foi estimada a probabilidade de o domicílio estar em condição de segurança alimentar ou insegurança alimentar leve (os dois primeiros níveis da EBIA), em contraponto a estar em situação de insegurança alimentar moderada ou grave. Os resultados do modelo estimado estão dispostos na Tabela 4.

O modelo mostrou-se bem ajustado. A estatística qui-quadrado de Hosmer-Lemeshow atestou a boa especificação do modelo, assim como resultou um percentual de classificação correto em $92,2 \%$, e uma área ROC de 0,80 - todos são parâmetros bastante razoáveis para esse tipo de modelo. ${ }^{3}$

2 Para esse modelo foi utilizada a implementação em Stata desenvolvida por Williams (2006), chamada de gologit2.

3 Detalhes sobre essas estatísticas podem ser consultados em Cameron e Trivedi (2010). Para 
E os resultados gerais obtidos estão alinhados com aqueles já observados em outros estudos similares, como em Hoffmann (2008; 2013), e também com as expectativas a partir da teoria.

Os resultados desse modelo binário sinalizam que a renda domiciliar per capita, utilizada de forma categorizada com base no salário mínimo da época, possui impacto direto significativo sobre a probabilidade de melhores condições de segurança alimentar. Domicílios com RDPC entre um e dois salários mínimos, por exemplo, têm 5,7 vezes mais chance de gozarem de segurança alimentar ou apenas insegurança leve.

Tabela 4 Modelo logit para probabilidade de segurança alimentar e insegurança leve Brasil, 2013

\begin{tabular}{lr|r|r}
\hline & $\begin{array}{r}\text { Coefi- } \\
\text { ciente }\end{array}$ & $\begin{array}{r}\text { Erro } \\
\text { padrão }\end{array}$ & $\begin{array}{r}\text { Odds } \\
\text { ratio }\end{array}$ \\
\hline Região & & & \\
\hline Sudeste (referência) & $-0,5001^{*}$ & 0,0503 & 0,6065 \\
\hline Nordeste & $-0,5215^{*}$ & 0,0599 & 0,5936 \\
\hline Norte & $-0,1969^{*}$ & 0,0628 & 0,8212 \\
\hline Sul & $-0,0948$ & 0,0738 & 0,9095 \\
\hline Centro-Oeste & $-0,3774^{*}$ & 0,0575 & 0,6856 \\
\hline Área urbana & $0,2126^{*}$ & 0,0361 & 1,2369 \\
\hline Gênero (masculino) & $-0,3632^{*}$ & 0,0324 & 0,6955 \\
\hline Raça (não branca) & $0,0076^{*}$ & 0,0012 & 1,0076 \\
\hline Idade & $0,0740^{*}$ & 0,0041 & 1,0768 \\
\hline Anos de estudo & & & \\
\hline Tipo de família & & & \\
\hline Casal sem filhos (referência) & $-0,1677^{*}$ & 0,0460 & 0,8456 \\
\hline Casal com todos os filhos menores de 14 anos & $0,8294^{*}$ & 0,0518 & 2,2919 \\
\hline Casal com todos os filhos de 14 anos ou mais & $0,3602^{*}$ & 0,0490 & 1,4337 \\
\hline Casal com filhos menores de 14 anos e de 14 anos ou mais & $0,8062^{*}$ & 0,0599 & 2,2393 \\
\hline Mãe com todos os filhos menores de 14 anos & $0,3329^{*}$ & 0,0768 & 1,3951 \\
\hline Mãe com todos os filhos de 14 anos ou mais & 0,0782 & 0,0616 & 1,0814 \\
\hline Mãe com filhos menores de 14 anos e de 14 anos ou mais & $0,3723^{*}$ & 0,0957 & 1,4511 \\
\hline Outros tipos de família & & & \\
\hline & & & \\
\hline
\end{tabular}

alguns testes o modelo precisou ser estimado sem considerar a amostra complexa, em função de sua implementação no Stata, sem maiores impactos sobre as conclusões. 
Tabela 4 (continuação)

\begin{tabular}{|c|c|c|c|}
\hline & $\begin{array}{l}\text { Coefi- } \\
\text { ciente }\end{array}$ & $\begin{array}{r}\text { Erro } \\
\text { padrão }\end{array}$ & $\begin{array}{l}\text { Odds } \\
\text { ratio }\end{array}$ \\
\hline \multicolumn{4}{|l|}{ Ocupação } \\
\hline \multicolumn{4}{|c|}{ Com carteira ou emprego formal (referência) } \\
\hline Desocupado & $-0,2153^{*}$ & 0,0501 & 0,8063 \\
\hline Sem carteira & $-0,4484^{*}$ & 0,0488 & 0,6386 \\
\hline Conta própria & $-0,2600^{*}$ & 0,0485 & 0,7711 \\
\hline Empregador & 0,3520 & 0,1865 & 1,4220 \\
\hline Não remunerado & 0,0833 & 0,2883 & 1,0868 \\
\hline Próprio consumo/uso & $-0,3638^{*}$ & 0,0887 & 0,6950 \\
\hline \multicolumn{4}{|l|}{ Atividade do domicílio } \\
\hline \multicolumn{4}{|c|}{ Apenas agrícola (referência) } \\
\hline Pluriativo & $-0,1230$ & 0,0698 & 0,8842 \\
\hline Não agrícola & 0,0716 & 0,0565 & 1,0743 \\
\hline Desocupado & 0,0823 & 0,0684 & 1,0857 \\
\hline \multicolumn{4}{|l|}{ RDPC (R\$) } \\
\hline \multicolumn{4}{|l|}{ Até 169,50 (referência) } \\
\hline$(169,50-339,00]$ & $0,5258^{*}$ & 0,0411 & 1,6918 \\
\hline$(339,00-678,00]$ & $1,0949 *$ & 0,0443 & 2,9890 \\
\hline$(678,00-1.356,00]$ & $1,7564^{*}$ & 0,0573 & 5,7914 \\
\hline$(1.356,00-2.034,00]$ & $2,4468^{*}$ & 0,1096 & 11,5515 \\
\hline$(2.034,00-3.390,00]$ & $2,7637^{*}$ & 0,1641 & 15,8587 \\
\hline Mais de $3.390,00$ & $4,6731^{*}$ & 0,4575 & 107,0238 \\
\hline Pessoas por cômodo & $-0,5855^{*}$ & 0,0447 & 0,5568 \\
\hline \multicolumn{4}{|l|}{ Índice de habitação } \\
\hline \multicolumn{4}{|l|}{ Até um item (referência) } \\
\hline Dois itens & $0,4076^{*}$ & 0,0683 & 1,5032 \\
\hline Três itens & $0,6055^{*}$ & 0,0756 & 1,8321 \\
\hline Constante & $1,0333^{*}$ & 0,1291 & 2,8104 \\
\hline
\end{tabular}

Fonte: Estimativas dos autores.

* Coeficiente significativo a $1 \%$.

Com relação ao tipo de família, casal ou mãe com filhos apresentam melhores chances de estarem em segurança alimentar ou insegurança leve quando comparados à situação de casais sem filhos (referência, no modelo). A explicação para esse resultado passa pela dominância da renda. 
Quando o modelo é estimado apenas com o tipo de família como variável explicativa, a situação dos casais sem filhos é a melhor; quando a renda é inserida, o resultado se inverte. Isso pode estar associado, entre outras questões relacionadas às demais variáveis, aos programas assistenciais que levam em consideração a presença de crianças em casa, como é o caso do Bolsa Família.

Os resultados também apontam que a estabilidade na renda do domicílio é importante. Em relação à pessoa de referência ter emprego com carteira assinada ou emprego formal, apenas a situação de empregador oferece melhores resultados em termos de probabilidade de segurança alimentar. Estar desocupado, ${ }^{4}$ trabalhando sem carteira assinada, por conta própria ou para o próprio consumo, quando em relação à estabilidade de um contrato de trabalho, tem impacto negativo estatisticamente significativo. Já as condições de atividade das pessoas do domicílio não se mostraram significativas.

Estar em área urbana tende a piorar a situação alimentar dos domicílios, assim como estar em outras regiões do Brasil que não seja o Sudeste. Ter como pessoa de referência um homem e/ou uma pessoa educada, também aumenta a probabilidade de boas condições de alimentação, assim como ser de raça branca - resultados que têm relação com aqueles já apontados por Hoffmann (2008; 2013).

Os resultados desse modelo, portanto, apontam para renda, tipo de família, emprego, educação e regionalização como importantes determinantes de insegurança alimentar. No entanto, a complexidade informacional da EBIA está inevitavelmente subestimada, uma vez que os diferentes graus de insegurança alimentar possivelmente estejam relacionados com diferentes fatores determinantes - ou, pelo menos, associados a pesos diferentes.

Para avançar no entendimento da insegurança alimentar incorporando as informações que as categorias da EBIA podem oferecer, estimamos o modelo logístico ordenado apresentado na Tabela 5.

Conforme já apresentado na metodologia, esse modelo considera todas as categorias da EBIA, mas mantém únicos os coeficientes das variáveis explicativas. $O$ que permite o cálculo de probabilidades para cada categoria são os diferentes coeficientes lineares (cut-offs). A interpretação dos 4 Aqui, desocupado não significa necessariamente desempregado. Aposentados que não têm trabalho se enquadram como desocupados. 
coeficientes é ligeiramente diferente: lê-se o valor como o impacto sobre a probabilidade de estar em categorias mais elevadas, partindo da categoria de base. No caso deste trabalho, trata-se da probabilidade de estar em níveis de situação melhor do que insegurança grave.

Tabela 5 Modelo logístico ordenado para as quatro categorias da EBIA - Brasil, 2013

\begin{tabular}{|c|c|c|c|}
\hline & $\begin{array}{l}\text { Coefi- } \\
\text { ciente }\end{array}$ & $\begin{array}{r}\text { Erro } \\
\text { padrão }\end{array}$ & $\begin{array}{l}\text { Odds } \\
\text { ratio }\end{array}$ \\
\hline \multicolumn{4}{|l|}{ Região } \\
\hline \multicolumn{4}{|l|}{ Sudeste (referência) } \\
\hline Nordeste & $-0,6334^{*}$ & 0,0417 & 0,5308 \\
\hline Norte & $-0,5792^{*}$ & 0,0509 & 0,5604 \\
\hline Sul & $-0,2039 *$ & 0,0446 & 0,8156 \\
\hline Centro-Oeste & $-0,1455^{* *}$ & 0,0582 & 0,8646 \\
\hline Área urbana & $-0,3187^{*}$ & 0,0441 & 0,7271 \\
\hline Gênero (masculino) & $0,2233^{*}$ & 0,0252 & 1,2502 \\
\hline Raça (não branca) & $-0,3131^{*}$ & 0,0218 & 0,7312 \\
\hline Idade & $0,0068^{*}$ & 0,0009 & 1,0068 \\
\hline Anos de estudo & $0,0493^{*}$ & 0,0028 & 1,0505 \\
\hline \multicolumn{4}{|l|}{ Tipo de família } \\
\hline \multicolumn{4}{|l|}{ Casal sem filhos (referência) } \\
\hline Casal com todos os filhos menores de 14 anos & $0,2082^{*}$ & 0,0320 & 1,2315 \\
\hline Casal com todos os filhos de 14 anos ou mais & $0,0942^{*}$ & 0,0312 & 1,0987 \\
\hline Casal com filhos menores de 14 anos e de 14 anos ou mais & $0,3252^{*}$ & 0,0381 & 1,3843 \\
\hline Mãe com todos os filhos menores de 14 anos & $-0,0248$ & 0,0496 & 0,9755 \\
\hline Mãe com todos os filhos de 14 anos ou mais & $-0,0590$ & 0,0398 & 0,9427 \\
\hline Mãe com filhos menores de 14 anos e de 14 anos ou mais & $-0,0009$ & 0,0622 & 0,9991 \\
\hline Outros tipos de família & $-0,0840^{*}$ & 0,0316 & 0,9195 \\
\hline \multicolumn{4}{|l|}{ Ocupação } \\
\hline \multicolumn{4}{|l|}{ Com carteira ou emprego formal (referência) } \\
\hline Desocupado & $-0,0966^{*}$ & 0,0320 & 0,9079 \\
\hline Sem carteira & $-0,3161^{*}$ & 0,0294 & 0,7290 \\
\hline Conta própria & $-0,1299 *$ & 0,0281 & 0,8782 \\
\hline Empregador & $0,2122^{*}$ & 0,0771 & 1,2364 \\
\hline Não remunerado & 0,2459 & 0,1683 & 1,2788 \\
\hline Próprio consumo/uso & $-0,3183^{*}$ & 0,0682 & 0,7274 \\
\hline
\end{tabular}


Tabela 5 (continuação)

\begin{tabular}{|c|c|c|c|}
\hline & $\begin{array}{l}\text { Coefi- } \\
\text { ciente }\end{array}$ & $\begin{array}{r}\text { Erro } \\
\text { padrão }\end{array}$ & $\begin{array}{l}\text { Odds } \\
\text { ratio }\end{array}$ \\
\hline \multicolumn{4}{|l|}{ Atividade do domicílio } \\
\hline \multicolumn{4}{|c|}{ Apenas agrícola (referência) } \\
\hline Pluriativo & $-0,1549 *$ & 0,0465 & 0,8565 \\
\hline Não agrícola & 0,0770 & 0,0445 & 1,0800 \\
\hline Desocupado & $0,1626^{*}$ & 0,0507 & 1,1766 \\
\hline \multicolumn{4}{|l|}{ RDPC (R\$) } \\
\hline \multicolumn{4}{|l|}{ Até 169,50 (referência) } \\
\hline$(169,50-339,00]$ & $0,4763^{*}$ & 0,0317 & 1,6101 \\
\hline$(339,00-678,00]$ & $0,9510 *$ & 0,0337 & 2,5884 \\
\hline$(678,00-1.356,00]$ & $1,4879 *$ & 0,0397 & 4,4278 \\
\hline$(1.356,00-2.034,00]$ & $1,9643^{*}$ & 0,0588 & 7,1297 \\
\hline$(2.034,00-3.390,00]$ & $2,4049 *$ & 0,0780 & 11,0774 \\
\hline Mais de $3.390,00$ & $3,0709 *$ & 0,1206 & 21,5609 \\
\hline Pessoas por cômodo & $-0,6105^{*}$ & 0,0343 & 0,5431 \\
\hline \multicolumn{4}{|l|}{ Índice de habitação } \\
\hline \multicolumn{4}{|l|}{ Até um item (referência) } \\
\hline Dois itens & $0,3663^{*}$ & 0,0587 & 1,4424 \\
\hline Três itens & $0,5433^{*}$ & 0,0631 & 1,7217 \\
\hline \multicolumn{4}{|l|}{ Cutoff's } \\
\hline /cut1 & $-2,5078^{*}$ & 0,1012 & \\
\hline /cut2 & $-1,4822^{*}$ & 0,1007 & \\
\hline /cut3 & $-0,0167$ & 0,1004 & \\
\hline
\end{tabular}

Fonte: Estimativas dos autores.

${ }^{*}$ Coeficiente significativo a $1 \%$. ${ }^{* *}$ Coeficiente significativo a $5 \%$.

Em linhas gerais, no que toca aos coeficientes $\beta$, os resultados são bastante similares àqueles obtidos com o modelo logístico binário. A única diferença que chama atenção é a redução, ainda que marginal, na dominância da renda e o fato de que duas categorias de atividade dos domicílios (pluriativos e desocupados) passam a ser significativas.

Os resultados ganham maior intuição e justificam a aplicação desse tipo de modelo, quando se estimam as probabilidades para cada uma das classes da variável dependente, condicionados ao comportamento das variáveis explicativas. No entanto, isso só é viável se o modelo logístico or- 
denado estimado respeitar a hipótese do paralelismo. O teste de Brant, na Tabela 6 , mostra que o modelo estimado viola essa hipótese - o que pode ser constatado pela alta significância do teste na linha Total.

Tabela 6 Teste de paralelismo de Brant*

\begin{tabular}{|c|c|c|c|}
\hline & Chi2 & $\mathrm{p}>\mathrm{Chi2}$ & GL \\
\hline Total & 1355,11 & 0,000 & 68 \\
\hline \multicolumn{4}{|l|}{ Região } \\
\hline \multicolumn{4}{|l|}{ Sudeste (referência) } \\
\hline Nordeste & 53,8900 & 0,0000 & 2,0 \\
\hline Norte & 0,8600 & 0,6500 & 2,0 \\
\hline Sul & 1,0200 & 0,5990 & 2,0 \\
\hline Centro-Oeste & 0,3000 & 0,8610 & 2,0 \\
\hline Área urbana & 15,9300 & 0,0000 & 2,0 \\
\hline Gênero (masculino) & 4,6300 & 0,0990 & 2,0 \\
\hline Raça (não branca) & 7,1000 & 0,0290 & 2,0 \\
\hline Idade & 4,2600 & 0,1190 & 2,0 \\
\hline Anos de estudo & 96,7300 & 0,0000 & 2,0 \\
\hline \multicolumn{4}{|l|}{ Tipo de família } \\
\hline \multicolumn{4}{|l|}{ Casal sem filhos (referência) } \\
\hline Casal com todos os filhos menores de 14 anos & 254,5800 & 0,0000 & 2,0 \\
\hline Casal com todos os filhos de 14 anos ou mais & 41,0300 & 0,0000 & 2,0 \\
\hline Casal com filhos menores de 14 anos e de 14 anos ou mais & 96,4300 & 0,0000 & 2,0 \\
\hline Mãe com todos os filhos menores de 14 anos & 39,3600 & 0,0000 & 2,0 \\
\hline Mãe com todos os filhos de 14 anos ou mais & 9,4300 & 0,0090 & 2,0 \\
\hline Mãe com filhos menores de 14 anos e de 14 anos ou mais & 38,9600 & 0,0000 & 2,0 \\
\hline Outros tipos de família & 53,2100 & 0,0000 & 2,0 \\
\hline \multicolumn{4}{|l|}{ Ocupação } \\
\hline \multicolumn{4}{|l|}{ Com carteira ou emprego formal (referência) } \\
\hline Desocupado & 16,4200 & 0,0000 & 2,0 \\
\hline Sem carteira & 7,9800 & 0,0190 & 2,0 \\
\hline Conta própria & 14,3000 & 0,0010 & 2,0 \\
\hline Empregador & 5,6900 & 0,0580 & 2,0 \\
\hline Não remunerado & 2,5700 & 0,2770 & 2,0 \\
\hline Próprio consumo/uso & 0,1400 & 0,9320 & 2,0 \\
\hline
\end{tabular}


Tabela 6 (continuação)

\begin{tabular}{lrrr}
\hline & Chi2 & p>Chi2 & GL \\
\hline Atividade do domicílio & & & \\
\hline Apenas agrícola (referência) & & & \\
\hline Pluriativo & 2,6300 & 0,2690 & 2,0 \\
\hline Não agrícola & 0,7500 & 0,6860 & 2,0 \\
\hline Desocupado & 6,0100 & 0,0500 & 2,0 \\
\hline RDPC (R\$) & & & \\
\hline Até 169,50 (referência) & & & \\
\hline (169,50 - 339,00] & 34,8800 & 0,0000 & 2,0 \\
\hline (339,00 - 678,00] & 65,3800 & 0,0000 & 2,0 \\
\hline (678,00 - 1.356,00] & 87,4000 & 0,0000 & 2,0 \\
\hline (1.356,00 - 2.034,00] & 47,4900 & 0,0000 & 2,0 \\
\hline (2.034,00 - 3.390,00] & 14,9900 & 0,0010 & 2,0 \\
\hline Mais de 3.390,00 & 17,3000 & 0,0000 & 2,0 \\
\hline Pessoas por cômodo & 2,4500 & 0,2940 & 2,0 \\
\hline Índice de habitação & & & \\
\hline Até um item (referência) & & & \\
\hline Dois itens & 6,8300 & 0,0330 & 2,0 \\
\hline Três itens & 3,7300 & 0,1550 & 2,0 \\
\hline & & &
\end{tabular}

Fonte: Estimativas dos autores.

* Resultados significativos indicam violação da hipótese do paralelismo.

Os resultados por variável não devem ser lidos como definitivos de que aquela variável viola a hipótese isoladamente, pois se trata do resultado do conjunto de variáveis. De qualquer modo, é um bom indicativo. Procedemos a testes eliminando diferentes conjuntos de variáveis que inspiravam suspeitas de influência mais direta, como é o caso da renda e variáveis pessoais. No entanto, o modelo continuou violando essa hipótese fundamental - e, se considerássemos manter apenas aquelas que o teste de Brant sugere como viáveis, o modelo ficaria demasiadamente incompleto e incorrendo em erros de especificação.

Esse resultado do teste de Brant sugere que há efeitos assimétricos para diferentes variáveis em relação às classes de insegurança alimentar da EBIA. Assim, passamos para a última abordagem sugerida neste trabalho, que é o modelo logístico generalizado. Esse modelo estima três equações simultaneamente (uma a menos que o total de categorias da EBIA). Para 
cada variável é testada a hipótese de igualdade dos coeficientes entre as categorias. Caso essa hipótese seja violada, diferentes coeficientes são estimados, sendo que a interpretação é a seguinte: o coeficiente estimado para cada equação indica a probabilidade de estar em categoria superior àquela. Veja resultados na Tabela 7.

A primeira observação a ser feita é que poucas variáveis respeitaram a hipótese do paralelismo e garantiram coeficiente único - em linha com o indicativo do teste de Brant. Estar nas regiões Norte, Sul e Centro-Oeste diminui na mesma proporção a probabilidade de melhorar os níveis de segurança alimentar, independentemente do ponto de partida. $\bigcirc$ mesmo ocorre com as variáveis de situação censitária, gênero e raça, mantendo as interpretações já destacadas no modelo logit tradicional da Tabela 4.

Quando a ocupação da pessoa de referência é empregador, não remunerado ou para próprio consumo ou uso, a probabilidade de melhora também é constante ao longo das diferentes categorias de insegurança alimentar. Com relação às atividades, o impacto de todas as categorias testadas se mostrou significativo e constante, sendo que domicílios pluriativos têm menores chances de incrementar a situação alimentar comparativamente aos domicílios em atividade apenas agrícola. Esse resultado tem respaldo no trabalho de Hoffmann e Kageyama (2007): os autores argumentam que isso acontece porque essa atividade incrementa a renda dos domicílios, que seria uma variável dominante no modelo.

Por fim, o número de pessoas por cômodos e o alcance de três itens nas condições de habitação também são características que oferecem resultado constante, em termos de probabilidade de o domicílio melhorar sua situação alimentar. $\bigcirc$ resultado é bastante intuitivo: sinal negativo para a primeira, e positivo para a segunda variável.

Para as demais variáveis, a assimetria dos efeitos indica que as probabilidades de gozar de melhores condições alimentares se alteram conforme a situação vigente no domicílio. Ainda que as diferenças não sejam grandes em termos de razão de chances (odds ratio), elas podem indicar comportamentos que, no conjunto, potencialmente remetam a conclusões importantes em termos de política pública. 


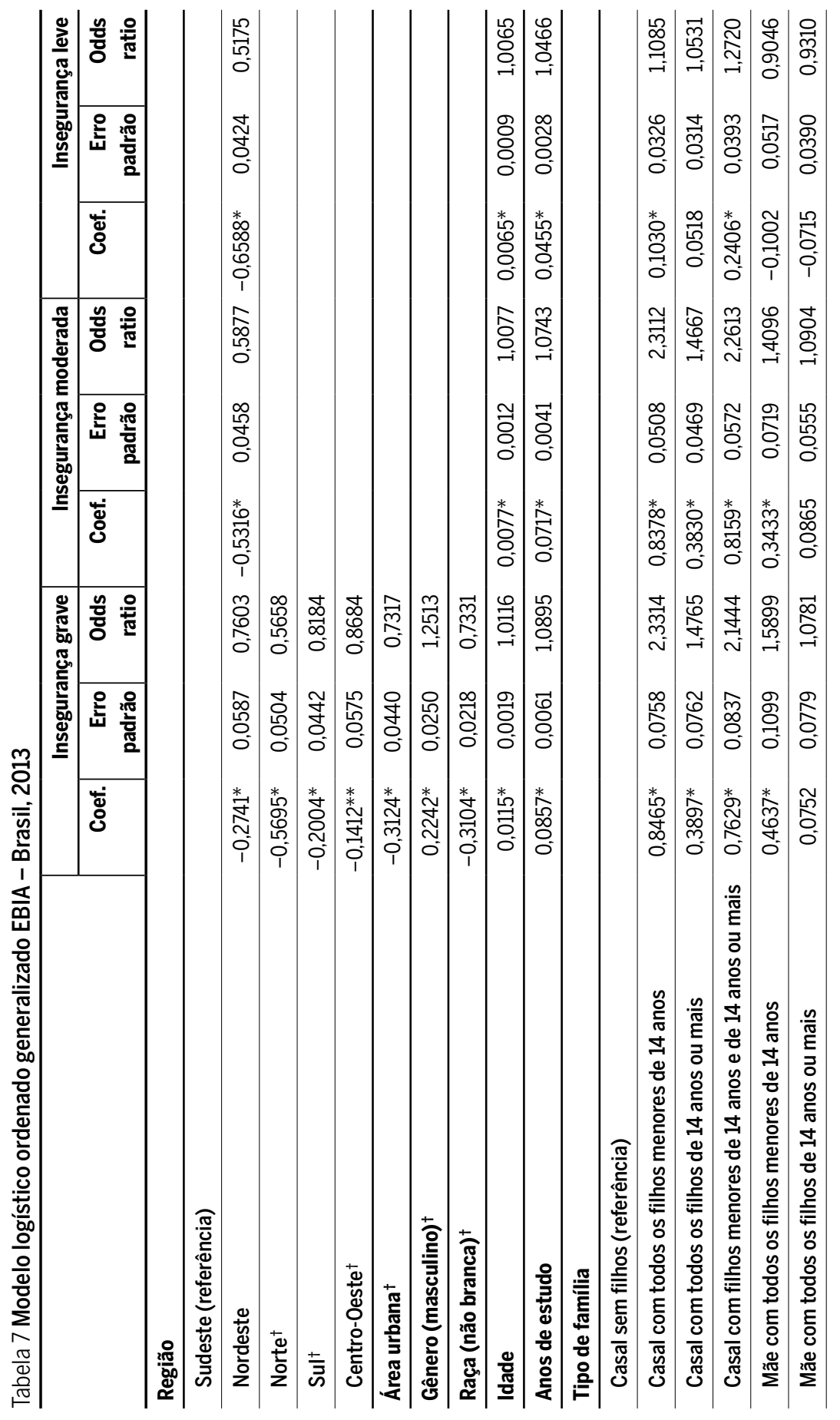




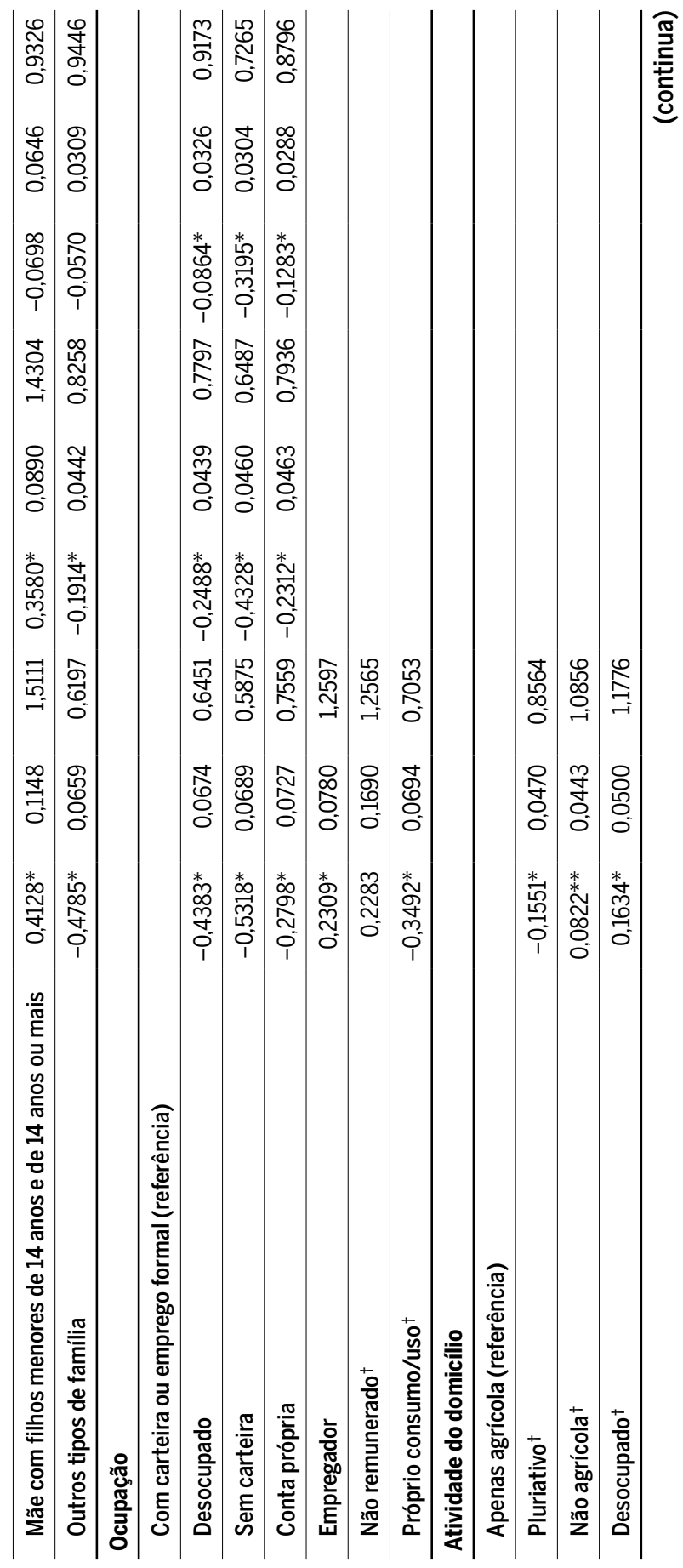




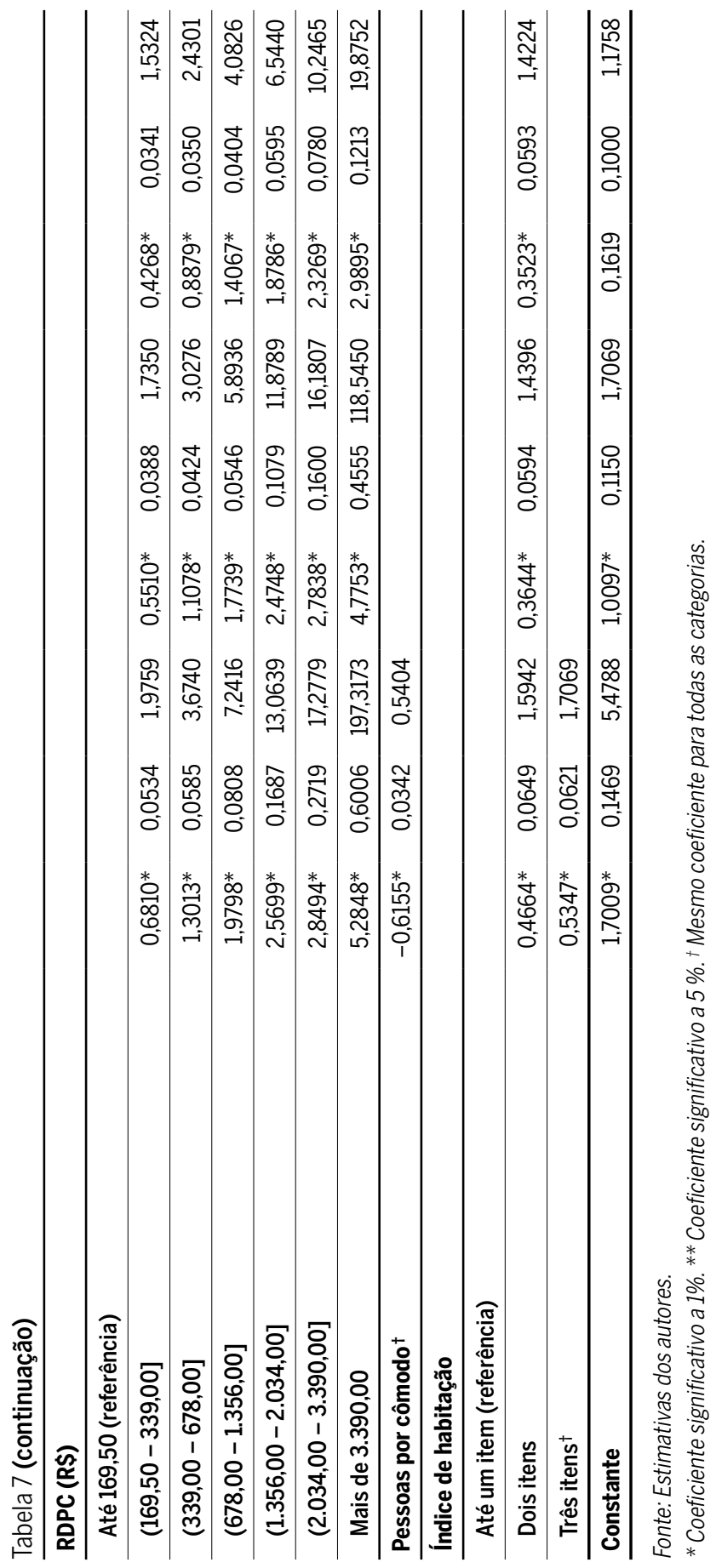


Estar na região Nordeste, na comparação com a região Sudeste, reduz em $24 \%$ a chance de o domicílio estar em melhores condições de alimentação quando se está em condição de insegurança alimentar grave. No entanto, considerando uma condição de insegurança alimentar leve, a chance de alcançar a segurança alimentar é reduzida em $48 \%$ quando nessa região. Ou seja, é cumulativamente mais difícil galgar melhores níveis de segurança alimentar estando na região Nordeste do Brasil.

Uma possível explicação para essa situação observada para o Nordeste é o relativo isolamento geográfico de municípios nessa região, como ressaltado por Gubert et al. (2017a). Corsi et al. (2017), por exemplo, observam a existência de uma associação positiva entre segurança alimentar e a facilidade de acesso ao mercado para pequenos agricultores no Chade.

Para os anos de estudo e idade, de fato existe diferença na probabilidade de migrar para condições de segurança alimentar melhores de acordo com a condição alimentar inicial: quanto mais severa a condição inicial, maior é o impacto direto da variável. No entanto, a diferença de resultados é muito pequena, tendo pouca representatividade analítica.

Sobre os tipos de família, os sinais apontam nas mesmas direções já indicadas no modelo logit tradicional. No entanto, cabe observar que a importância do tipo de arranjo familiar cai significativamente quando o domicílio alcança o nível de insegurança leve. Trata-se de um indicativo de que a partir desse nível a situação toma contornos mais complexos, com a estrutura familiar perdendo importância relativa na explicação.

Com relação às ocupações, estar desocupado, sem carteira ou por conta própria, em relação a estar ocupado com carteira assinada ou emprego formal, tem efeito negativo sobre a probabilidade de melhores condições alimentares. Porém, quanto mais os domicílios se afastam de uma condição de insegurança alimentar grave, menos importante se torna este impacto. Esse efeito pode estar relacionado a dois fatores. $O$ primeiro deles é que a minoria dos domicílios está em insegurança alimentar moderada ou grave, de modo que contempla fundamentalmente uma parcela da população consideravelmente miserável, com difícil inserção no mercado de trabalho, o que torna a situação desses domicílios ainda mais delicada.

Outro fator é que uma vez alçadas a melhores condições, as famílias são melhor atendidas por programas sociais e também têm melhores condições de buscar sustento, mesmo que de maneira informal. Mensagem-chave nessa questão, portanto: atenção especial para capacitação e 
inserção profissional de pessoas em situação de insegurança alimentar moderada e grave.

A renda domiciliar per capita demonstrou o comportamento esperado: quanto mais elevada, melhor a resposta em termos de condições de alimentação. No entanto, a abordagem desse modelo ordenado generalizado oferece uma mensagem mais elaborada. Podemos verificar, por exemplo, que garantir entre um quarto e meio salário mínimo domiciliar per capita praticamente dobra as chances de os domicílios em situação de insegurança alimentar grave melhorarem (odds ratio de 1,97); porém, o efeito quando a situação é insegurança leve é apenas a metade disso para fazer que o domicílio goze de segurança alimentar (odds ratio de 1,53).

$\mathrm{Na}$ faixa entre meio e um salário mínimo domiciliar per capita, as chances de obter segurança alimentar aumentam em 143\% - entre um e dois salários mínimos domiciliar per capita, as chances são de mais de 300\% maiores. Ou seja, a renda tem importante papel entre os fatores condicionantes estudados, sendo válido observar seu comportamento assimétrico nas faixas mais baixas. Esse resultado é de particular interesse em função de políticas públicas como o Bolsa Família, que realiza complementação de renda adicional para famílias em situação de extrema pobreza.

Esse tipo de modelo oferece também a possiblidade de estimar os efeitos marginais de uma variável - ou uma combinação delas - sobre a probabilidade de ocorrência de cada uma das categorias da variável dependente, mantendo-se tudo o mais constante. Por restrição de espaço e pelo fato de que o objetivo central deste estudo é apresentar as potencialidades dessa abordagem no debate sobre insegurança alimentar, não avançaremos nessa direção. A seção seguinte traz as nossas conclusões.

\section{Conclusões}

Este trabalho trata de um tema bastante sensível para países em desenvolvimento, como o Brasil. Ainda que avanços tenham sido observados ao longo das últimas duas décadas, a segurança alimentar do país não pode ser considerada como garantida. Nesse sentido, os estudos sobre esse assunto ainda são demandados e fundamentais para o entendimento desse fenômeno.

A complexidade envolvida em medidas de segurança alimentar como a EBIA oferece uma série de oportunidades analíticas. Este artigo buscou ve- 
rificar como um grupo de características sociodemográficas afeta a probabilidade de ocorrência de diferentes níveis de insegurança alimentar. Nesse sentido, o artigo avançou ao incorporar a complexidade de informações proporcionadas pela EBIA em diferentes modelos de probabilidade que validassem a hipótese central de efeitos assimétricos das variáveis explicativas em relação às diferentes categorias de insegurança alimentar da EBIA.

O modelo de probabilidade generalizado mostrou-se o mais adequado e robusto nas estimações. Os resultados apontaram para o fato de que existe assimetria nos efeitos de vários determinantes da insegurança alimentar de acordo com a situação vigente no momento. Ou seja, ficou demonstrado, por exemplo, que a influência de ocupação, atividade e renda, por exemplo, tem magnitudes de impacto diferentes para domicílios em insegurança grave, moderada ou leve. Isto é, o fato de um domicílio estar sujeito à ou imerso em uma situação de insegurança alimentar em algum grau depende, sobretudo, das suas características sociodemográficas.

Ainda que as diferenças encontradas não sejam altas, em termos absolutos, elas sugerem que políticas públicas devem tomar essas conclusões em consideração. Diferentes ações e cuidados para as diversas situações de insegurança alimentar e seus determinantes podem fazer diferença em termos de resultados das políticas públicas.

\section{Referências}

AGBOOLA, M. O.; BALCILAR, M. Impact of food security on urban poverty: A case study of Lagos State, Nigeria. Procedia-Social and Behavioral Sciences, v. 62, p. 1.225-1.229, 2012.

ATTANASIO, O.; DI MARO, V.; LECHENE, V.; PHILLIPS, D. Welfare consequences of food prices increases: Evidence from rural Mexico. Journal of Development Economics, v. 104, p. 136-151, 2013.

BARRETT, C. B. Measuring food insecurity. Science, v. 327, n. 5.967, p. 825-828, 2010.

BELIK, W. 2003. Perspectivas para segurança alimentar e nutricional no Brasil. Saúde Soc. v. 12 , n. 1, p. 12-20, 2003.

BHALLA, G.; HANDAB, S.; ANGELESC, G.; SEIDENFELDD, D. The effect of cash transfers and household vulnerability on food security in Zimbabwe. Food Policy, v. 74, p. 82-99, 2018.

BICKEL, G.; NORD, M.; PRICE, C.; HAMILTON, W.; COOK, J. Guide to measuring household food security, Revised 2000. U.S. Department of Agriculture, Food and Nutrition Service, Alexandria VA, 2000. 
BRANT, R. Assessing proportionality in the proportional odds model for ordered logistic regression. Biometrics, v. 46, n. 4, p. 1.171-1.178, 1990.

BURCHI, F.; DE MURO, P. From food availability to nutritional capabilities: Advancing food security analysis. Food Policy, v. 60, p. 10-19, 2016.

CAMERON, A.C.; TRIVEDI, P.K. Microeconometrics using Stata. USA: Stata Press, 2010.

CHAMBERS, R.; CONWAY, G. R. Sustainable rural livelihoods: Practical concepts for the $21^{\text {st }}$ century. Institute for Development Studies, IDS Discussion Paper number 296, 1991.

COlEMAN-JENSEN, A.; RABBITT, M. P.; GREGGORY, C. A.; SINGH, A. Household Food Security in the United States in 2015. Economic Research Report Number 215, 2016.

CORRÊA, A. M. S. Insegurança alimentar medida a partir da percepção das pessoas. Estudos Avançados, v. 21, n. 60, p. 143-154, 2007.

CORSI, S.; MARCHISIO, L. V.; ORSI, L. Connecting smallholder farmers to local markets: Drivers of collective action, land tenure and food security in East Chad. Land Use Policy, v. 68, p. 39-47, 2017.

DEVEREUX, S.; BERGE, K. Famine in the twentieth century. Brighton: Institute of Development Studies, 2000.

ELLIS, F. Household strategies and rural livelihood diversification. The Journal of Development Studies, v. 35, n. 1, p. 1-38, 1998.

ELLIS, F. Rural livelihoods and diversity in developing countries. Oxford University Press, 2000.

ELLIS, F.; BIGGS, S. Evolving themes in Rural Development 1950s-2000s. Development Policy Review, v. 19, n. 4, p. 437-448, 2001.

FAO; IFAD; UNICEF; WFP and WHO. The State of Food Security and Nutrition in the World 2017. Building resilience for peace and food security. Rome, FAO, 2017.

FÁVARO, T.; RIBAS, D. L. B.; ZORZATTO, J. R.; SEGALL-CORRÊA, A. M., \& PANIGASSI, G. Segurança alimentar em famílias indígenas terena, Mato Grosso do Sul, Brasil. Cadernos de Saúde Pública, v. 23, p. 785-793, 2007.

FU, V. Sg88: Estimating generalized ordered logit models. Stata technical bulletin, v. 44, n. 8, p. 160-164, 1998.

GUBERT, M. B.; SANTOS, S. M. C.; SANTOS, L. M. P.; PÉREZ-ESCAMILLA, R. A municipallevel analysis of secular trends in severe food insecurity in Brazil between 2004 and 2013. Global Food Security, v. 14, p. 61-67, 2017.

HIDROBO, M.; HODDINOTT, J.; PETERMAN, A.; MARGOLIES, A.; MOREIRA, V. Cash, food, or vouchers? Evidence from a randomized experiment in northern Ecuador. Journal of Development Economics, v. 107, p. 144-156, 2014.

HOFFMANN, R. Determinantes da insegurança alimentar no Brasil: análise dos dados da PNAD de 2004. Seg. Alim. Nutr., v. 15, n. 1, p. 49-61, 2008.

HOFFMANN, R. Determinantes da insegurança alimentar no Brasil em 2004 e 2009. Seg. Alim. Nutr., v. 20, n. 2, p. 219-235, 2013.

HOFFMANN, R.; KAGEYAMA, A. Pobreza, insegurança alimentar e pluriatividade no Brasil. Teoria e Evidência Econômica, v. 14, n. 29, p. 9-35, 2007. 
IBGE. Instituto Brasileiro de Geografia e Estatística. Pesquisa Nacional por Amostra de Domicílio: Segurança Alimentar. 2013.

IBGE. Instituto Brasileiro de Geografia e Estatística. Pesquisa Nacional por Amostra de Domicílio: Segurança Alimentar. 2014

IONESCU-ITTU, R.; GLYMOUR, M. M.; KAUFMAN, J. S. A difference-in-differences approach to estimate the effect of income-supplementation on food insecurity. Preventive Medicine, v. 70, p. 108-116, 2015.

JONES, A. D.; NGURE, F. M.; PELTO, G.; YOUNG, S. L. What are we assessing when we measure food security? A compendium and review of current metrics. Advances in Nutrition, v. 4, n. 5, p. 481-505, 2013.

MAITRA, C.; RAO, D. S. P. Poverty-Food Security Nexus: Evidence from a Survey of Urban Slum Dwellers in Kolkata. World Development, v. 72, p. 308-325, 2015.

MALLICK, D.; RAFI, M. Are female-headed households more food insecure? Evidence from Bangladesh. World Development, v. 38, n. 4, p. 593-605, 2010.

MALUF, R. S.; MENEZES, F.; VALENTE, F. L. Contribuição ao tema da segurança alimentar no Brasil. Cadernos de Debate, v. 4, p. 66-88, 1996.

MATTOS, E. J.; BAGOLIN, I. P. Reducing Poverty and Food Insecurity in Rural Brazil: the Impact of the Zero Hunger Program. Eurochoices, v. 16, n. 1, p. 43-49, 2017.

MAXWELL, S. Food security: A post-modern perspective. Food Policy, v. 21, n. 2, p. 155-170, 1996

MURAOKA, R.; JIN, S.; JAYNE, T. S. Land access, land rental and food security: Evidence from Kenya. Land Use Policy, v. 70, p. 611-622, 2018.

OXFAM. Fighting Hunger in Brazil. Oxfam Case Study. 2010.

OSMANI, S. R. The entitlement approach to famine: An assessment. UNU World Institute for Development Economics Research, Working Paper number 107, 1993.

PÉREZ-ESCAMILLA, R.; SEGALL-CORRÊA, A. M.; KURDIAN-MARANHA, L.; SAMPAIO, F; MARÍN-LEÓN, L.; PANIGASSI, G. An adapted version of the US Department of Agriculture Food Insecurity module is a valid tool for assessing household food insecurity in Campinas, Brazil. The Journal of nutrition, v. 134, n. 8, p. 1.923-1.928, 2004.

PÉREZ-ESCAMILLA, R.; SEGALL-CORRÊA, A. M. Food insecurity measurement and indicators. Revista de Nutrição, v. 21, p. 15s-26s. 2008.

ROCHA, C.; BURLANDY, L.; MAGALHÃES, R. (Org.). Segurança Alimentar e Nutricional: perspectivas, aprendizados e desafios para as políticas públicas. Rio de Janeiro: Editora Fiocruz, 2013.

SCOONES, I. Sustainable rural livelihoods a framework for analysis. Institute for Development Studies, IDS Working Paper number 72, 1998.

SEN, A. K. Poverty and famines: An essay on entitlement and deprivation. Oxford: Oxford University Press, 1981a.

SEN, A. K. Ingredients of famine analysis: Availability and entitlements. The Quarterly Journal of Economics, v. 96, n. 3, p. 433-464, 1981 b.

SEN, A. K. Desigualdade reexaminada. 2. ed. Rio de Janeiro: Record, 2008. 
SEN, A. K. Desenvolvimento como liberdade. São Paulo: Companhia das Letras, 2010.

SEN, A. K.; DRÈZE, J. Hunger and Public Action. Oxford: Clarendon Press, 1989.

SILVA, P. L. N.; PESSOAL, D. G. C.; LILA, M. F. Análise estatística de dados da PNAD: incorporando a estrutura do plano amostral. Ciências e Saúde Coletiva [on-line], v. 7, n. 4, p. 659-670, 2002.

SMITH, L. C.; OBEID, A. E.; JENSEN, H. H. The geography and causes of food insecurity in developing countries. Agricultural Economics, v. 22, n. 2, p. 199-215, 2000.

TAKAGI, M. Implantação da política de segurança alimentar e nutricional no Brasil: seus limites e desafios. Tese (Doutorado) - Instituto de Economia/Unicamp, Campinas, 2006.

VIANNA, R. P. T.; SEGALL-CORRÊA, A. M. Insegurança alimentar das famílias residentes em municípios do interior do estado da Paraíba, Brasil. Revista de Nutrição, v. 21, n. 10, p. 111-122, 2008.

WEBB, P.; COATES, J.; FRONGILlO, E. A.; ROGERS, B. L.; SWINDALE, A., BILINSKY, P. Measuring household food insecurity: Why it's so important and yet so difficult to do. The Journal of Nutrition, v. 136, n. 5, p. 1.404s-1.408s, 2006.

WILLIAMS, R. Generalized ordered logit/partial proportional odds models or ordinal dependent variables. Stata Journal, v. 6, n. 1, p. 58-82, 2006.

WILLIAMS, R. 2016. Understanding and interpreting generalized ordered logit models. The Journal of Mathematical Sociology, v. 40, n. 1, p. 7-20, 2016.

\section{Sobre os autores}

Ely José de Mattos - ely.mattos@pucrs.br Programa de Pós-Graduação em Economia (PPGE-PUCRS), Pontifícia Universidade Católica do Rio Grande do Sul, Porto Alegre, RS, Brasil.

ORCID: https://orcid.org/0000-0002-6364-1599.

Lorenzo Bianchi-lorenzolubi@hotmail.com

Programa de Pós-Graduação em Economia (PPGE-PUCRS), Pontifícia Universidade Católica do Rio Grande do Sul, Porto Alegre, RS, Brasil.

ORCID: https://orcid.org/0000-0002-0890-8086.

Camila Horst Toigo - camilahorstoigo@gmail.com

Programa de Pós-Graduação em Economia (PPGE-PUCRS), Pontifícia Universidade Católica do Rio Grande do Sul, Porto Alegre, RS, Brasil.

ORCID: https://orcid.org/0000-0002-1491-2508.

Este artigo contou com auxílio financeiro do CNPq.

\section{Sobre o artigo}

Recebido em 13 de fevereiro de 2019. Aprovado em 12 de fevereiro de 2020. 